\title{
Modeling and Characterization of Stochastic Resistive Switching in Single Ag2S Nanowires
}

\author{
Nikolay Frick ( $\nabla$ nvfrik@ncsu.edu ) \\ North Carolina State University \\ Mahshid Hosseini \\ North Carolina State University \\ Damien Guilbaud \\ North Carolina State University \\ Ming Gao \\ North Carolina State University \\ Thomas LaBean \\ North Carolina State University
}

\section{Research Article}

Keywords: Modeling and Characterization, Stochastic Resistive Switching, Single Ag2S Nanowires, metallic filaments, physical devices, memristive networks

Posted Date: December 13th, 2021

DOI: https://doi.org/10.21203/rs.3.rs-1096723/v1

License: (c) (i) This work is licensed under a Creative Commons Attribution 4.0 International License.

Read Full License 


\title{
Modeling and Characterization of Stochastic Resistive Switching in Single $\mathrm{Ag}_{2} \mathrm{~S}$ Nanowires
}

\author{
Nikolay Frick ${ }^{1,+}$, , Mahshid Hosseini ${ }^{2,1,+}$, Damien Guilbaud ${ }^{3}$, Ming Gao ${ }^{1}$, and Thomas \\ LaBean ${ }^{1, * *}$
}

${ }^{1}$ NC State University, Materials Science and Engineering, Raleigh, 27606, USA

${ }^{2}$ NC State University, Physics, Raleigh, 27606, USA

${ }^{3}$ NC State University, Biomedical Engineering, Raleigh, 27606, USA

*nvfrik@ncsu.edu

**thlabean@ncsu.edu

+these authors contributed equally to this work

\begin{abstract}
Chalcogenide resistive switches (RS), such as $\mathrm{Ag}_{2} \mathrm{~S}$, change resistance due to the growth of metallic filaments between electrodes along the electric field gradient. Therefore, they are candidates for neuromorphic and volatile memory applications. This work analyzed the RS of individual $\mathrm{Ag}_{2} \mathrm{~S}$ nanowires (NWs) and extended the basic RS model to reproduce experimental observations. The work models resistivity of the device as a percolation of the conductive filaments. It also addressed continuous fluctuations of the resistivity with a stochastic change in volume fractions of the filaments in the device. As a result, these fluctuations cause unpredictable patterns in current-voltage characteristics and include a spontaneous change in resistance of the device during the linear sweep that conventional memristor models with constant resistivity cannot represent. The parameters of the presented stochastic model of a single $\mathrm{Ag}_{2} \mathrm{~S} \mathrm{NW}$ fit the experimental data reproduced key features of $\mathrm{RS}$ in the physical devices. Moreover, the model suggested a non-core shell structure of the $\mathrm{Ag}_{2} \mathrm{~S}$ NWs. The outcome of this work is aimed to aid in simulating large self-assembled memristive networks and help to extend existing RS models.
\end{abstract}

\section{Introduction}

Resistive switching devices attract much interest due to potential applications in neuromorphic computing. Unlike conventional computing architectures, neuromorphic computers store and process data in one place, and therefore can perform massively parallel computations at low energy $\operatorname{cost}^{1-3}$ that is not constrained by the von Neumann bottleneck ${ }^{4}$.

Ionically conductive silver chalcogenides are one of the most attractive RS materials due to the simplicity of their production. Chalcogenides RS have been extensively studied ${ }^{5-10}$ and have already shown its potential use in a proof of concept neuromorphic applications such as arbitrary signal generation ${ }^{11}$, speech processing ${ }^{12}$, and decision-making devices ${ }^{13,14}$. Furthermore, the low cost and ease of large-scale production of the $\mathrm{Ag}_{2} \mathrm{~S}$ NWs offer a convenient way to manufacture neuromorphic computing devices through random self-assembly ${ }^{11,15}$. Moreover, $\mathrm{Ag}_{2} \mathrm{~S}$ NWs provide the possibility of manufacturing high-density 3D neuromorphic circuits ${ }^{16,17}$.

In silico simulation of the neuromorphic devices offers a convenient way to understand the properties of these materials. However, while the simulation of individual devices in cross-bar array neuromorphic architectures yields reproducible results ${ }^{18}$, reliable simulation of randomly assembled memristive networks has not been reported yet. Noise and unpredictable phase change in individual devices pose the main obstacles in simulating random and self-assembled neuromorphic devices. In particular, the characteristics of RS of $\mathrm{Ag}_{2} \mathrm{~S}$ NWs exhibits noise ${ }^{19,20}$ and nonlinear behavior that cannot be fully explained by a simple thin-film memristor model first proposed by Strukov et al. ${ }^{21}$.

Modeling of large RS nanowire networks, such as $\mathrm{Ag}_{2} \mathrm{~S}$ NW, can be improved by understanding the morphology of the material and its dynamic properties. There are several polymorphs of $\mathrm{Ag}_{2} \mathrm{~S}$ that exist in a narrow temperature range. For example, acanthite $\mathrm{Ag}_{2} \mathrm{~S}-\alpha$ is a low-temperature polymorph with a monoclinic crystal structure that is stable up to $\sim 450 \mathrm{~K}^{22}$. Above $450 \mathrm{~K}$ and up to $\sim 860 \mathrm{~K} \mathrm{Ag}_{2} \mathrm{~S}$ is in argentite $\mathrm{Ag}_{2} \mathrm{~S}-\beta$ phase with an ordered bcc lattice of sulfur atoms and $\mathrm{Ag}^{+}$ions that partially occupy tetrahedral and octahedral sites that gives it excellent ion mobility and increased electrical conductivity ${ }^{22-25}$.

Besides temperature, the transformation between acanthite and argentite can also be induced by an external electric field that displays hysteresis in the current vs. voltage ${ }^{5,24}$. However, unlike transition metal oxide RS devices ${ }^{26,27}$, the current in $\mathrm{Ag}_{2} \mathrm{~S}$ devices exhibit more considerable instability and noise related to the instability of Ag filaments and Joule heating ${ }^{6}$. It was recently reported that the noise in $\mathrm{Ag}_{2} \mathrm{~S}$ follows a 1/f pattern caused by dynamical point defects in the metallic filaments 
causing temporal instability ${ }^{19,20}$.

Scattered Ag nano-islands in a volume and on a surface of oxide ionic conductors were reported in other experimental configurations with Ag filaments serving as resistive switches. In particular, Ag clusters were observed on the surface of $\mathrm{ZnO}$ nanowire during $\mathrm{RS}$ cycles in an $\mathrm{Ag} / \mathrm{ZnO} / \mathrm{Pt}$ system $^{28}$. In another study, Wang et al. showed an in situ formation of $\mathrm{Ag}$ nanoclusters with HRTEM in a planar system of $\mathrm{Au} / \mathrm{SiO}_{\mathrm{x}} \mathrm{N}_{\mathrm{y}}: \mathrm{Ag} / \mathrm{Au}^{29}$. Other observations showed spontaneous protrusions of $\mathrm{Ag}$ nano-islands in the $\mathrm{Ag}_{2} \mathrm{~S}$ phase ${ }^{30}$ and under electric field bias ${ }^{13}$. Finally, a detailed study of $\mathrm{RS}_{\text {of }} \mathrm{Ag}_{2} \mathrm{~S}$ ionic conductors performed with HRTEM clarified the mechanism of RS in $\mathrm{Ag}_{2} \mathrm{~S}$ devices ${ }^{24}$.

The main contribution of this work is a stochastic model of RS of a single $\mathrm{Ag}_{2} \mathrm{~S} \mathrm{NW}$ that is based on single-wire measurements performed with a nanomanipulator under an optical microscope and is comparable to other reports that studied RS in chalcogenides. The model extends the basic RS model by Strukov et al. ${ }^{21}$ with the modification of resistivity of the device in the ON state and an assumption that resistivity of highly conductive state changes according to the laws of percolation theory. In particular, the variable $\mathrm{R}_{\mathrm{ON}}$ changes proportionally to the volume fraction of spontaneously created and destroyed metallic Ag nano-islands and conductive filaments between them in the mixture of $\mathrm{Ag}_{2} \mathrm{~S}-\alpha$ and $\mathrm{Ag}_{2} \mathrm{~S}-\beta$ phases.

The fitted model exhibited key features of the RS of single $\mathrm{Ag}_{2} \mathrm{~S}$ NW, such as a spontaneous change in resistivity expressed in IV loop twisting and reversal during the linear voltage sweep.

\section{Results}

The Ag NWs were produced with a simple polyol method ${ }^{31-33}$ and further sulfurized in a sulfur-rich ethanol (EtOH) suspension at different times (see methods). The surface morphology of the resulting $\mathrm{Ag}_{2} \mathrm{~S}$ NWs was examined by scanning electron microscopy (JEOL, JSM-6010LA ) as shown in Figure 1 (a) and high-resolution transmission electron microscopy (Talos F200X G2), as shown in Figures 1 (b) and (c). Before sulfur treatment, the mean length and diameter of Ag NWs were about $53 \mu \mathrm{m}$ and $128 \mathrm{~nm}$, respectively. In contrast, the sulfurization modified mechanical properties and surface appearance of the wires that reduced their length to average length to $25 \mu \mathrm{m}$ and increased diameter to $135 \mathrm{~nm}$, respectively.

Figure 1 (e) shows X-ray Diffraction (XRD) spectra of Ag NWs before sulfurization on top (inset shows the color of $\mathrm{Ag}$ NWs suspension), $\mathrm{Ag}$ NWs with partial $\mathrm{Ag}_{2} \mathrm{~S}$ inclusions when the suspension is brown in the middle frame, and black $\mathrm{Ag}_{2} \mathrm{~S}$ NWs at the bottom of the acanthite $\mathrm{Ag}_{2} \mathrm{~S}-\alpha$ phase. These results are comparable with Levard et al.'s XRD data of Ag nanoparticles sulfurization, which showed the existence of an additional phase that caused a change in intensity by modifying the ratio of $\mathrm{S} / \mathrm{Ag}^{34}$.

The Energy Dispersive X-Ray Spectroscopy (EDS) spectra and quantitative elemental data of different time intervals of AgNW exposure to sulfur are shown in Figure 1 (d-e). The color of the wires attests to the overall amount of the embedded sulfur and therefore expected conductivity, with light brown being the most conductive and dark brown and black being the least conductive ${ }^{35}$.

The schematic drawings in Figure 1 (d) have been designed based on the elemental weight percentage from the EDS and the SEM images. Since SEM collects data from the surface of the material, after a short reaction time when the suspension was still light brown (top), more $\mathrm{S}$ was on the surface of the wires. In contrast, as more time passed the color became darker, sulfur diffused deeper inside the bulk of wires gradually, and there were still Ag regions as well (middle). Hence, in the bottom frame, which illustrates the black suspension, there are islands of $\mathrm{Ag}$ and $\mathrm{Ag}_{2} \mathrm{~S}$ inside the bulk of the wires and still, some $\mathrm{S}$ particles on the wires' surface, which is consistent with observations in prior work ${ }^{13,24,30,36-39}$.

\subsection{Electrical Characterization}

Figure 2 shows the experimental setup for single wire measurements. In the setup, an electrochemically etched Platinum-Iridium $(\mathrm{Pt} / \mathrm{Ir})$ wire of $127 \mu \mathrm{m}$ diameter was composed of $20 \% \mathrm{wt}$. Ir (Alfa Aesar) microprobe was produced with an adopted method described by Zhang et al. ${ }^{40}$ and Khan et al. ${ }^{41}$. The Pt/Ir microprobe was installed on a nanomanipulator (Kleindiek MM3A) attached to the XY-stage of an optical microscope (Nikon Optiphot 100). A microscope slide was used as the insulator substrate, and Ag paint was applied on the substrate to make the reactive electrode while the Pt/Ir microprobe served as the inert electrode.

In the configuration, the Ag paint electrode is connected to a copper tape at one end, and the microprobe's tip on the dangling end forms a two-terminal device, as shown in Figure 2 (a). The system was connected to a source measure unit (SMU) (Keithley 2636B), with the "high" end connected to the tip of the nanoprobe and the "low" end through a 100 resistor to prevent damage. Simultaneously, the process was observed with a microscope at 1000x magnification and long working distance optics to navigate the tip of the nanomanipulator.

A few different lengths of the same wire were stimulated with one period of bipolar triangular voltage as shown in Figures 2 (b) and (c) correspondingly. First, the $20 \mu \mathrm{m}$ long $\mathrm{Ag}_{2} \mathrm{~S}$ NW was chosen, and the contact with the nanomanipulator's tip was established for two-probe IV characterization Figure 2 (b). Then the characteristics were measured with the same wire which length was mechanically reduced to $8 \mu \mathrm{m}$, Figure 2 (c). 

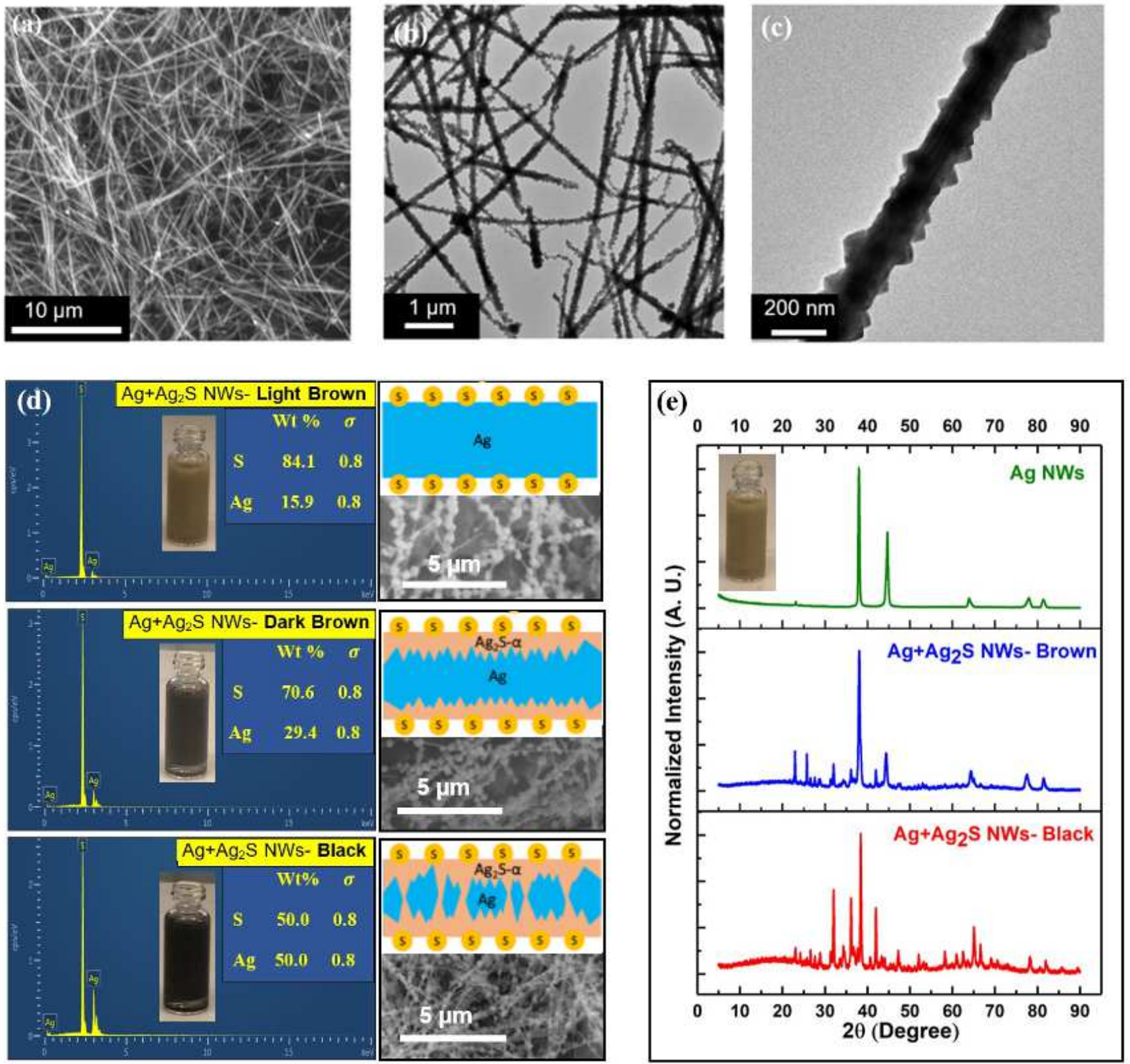

Figure 1. Characterization of produced $\mathrm{Ag}$ and $\mathrm{Ag}_{2} \mathrm{~S}$ NWs a) Scanning electron micrograph ( $\mathrm{SEM}$ ) of $\mathrm{Ag} 2 \mathrm{~S}$ NWs (when the suspension was dark brown). b) Transmission electron microscopy (TEM) of a network of $\mathrm{Ag}_{2} \mathrm{~S}$ NWs. c) TEM of a single $\mathrm{Ag}_{2} \mathrm{~S}$ NW. d) Energy Dispersive X-Ray Spectroscopy (EDS) spectra and quantitative elemental data from Ag NWs at different durations of exposure of Ag NW to sulfur. At the end of the first 5 minutes of exposure to S, the Ag NW suspension turned light brown (top). After 7 mins suspension turned dark brown (middle). Finally, with increased amounts of $\mathrm{S}$ diffused into the volume of the wire from the surface, the suspension turned black (bottom). On the right side, SEM images and schematic drawings of NWs correspond to each of the timesteps of sulfurization. Once Ag NWs were placed in the sulfur environment, the proportions of $\mathrm{Ag}$ nano-clusters in the $\mathrm{Ag}_{2} \mathrm{~S}$ matrix varied proportionally to the duration of exposure to sulfur. e) X-ray Diffraction (XRD) spectra of Ag NWs before sulfurization, the inset shows Ag NWs suspension color (top), Ag NWs with partial $\mathrm{Ag}_{2} \mathrm{~S}$ inclusions (brown; middle), and black $\mathrm{Ag}_{2} \mathrm{~S}$ NWs (bottom). 
a)

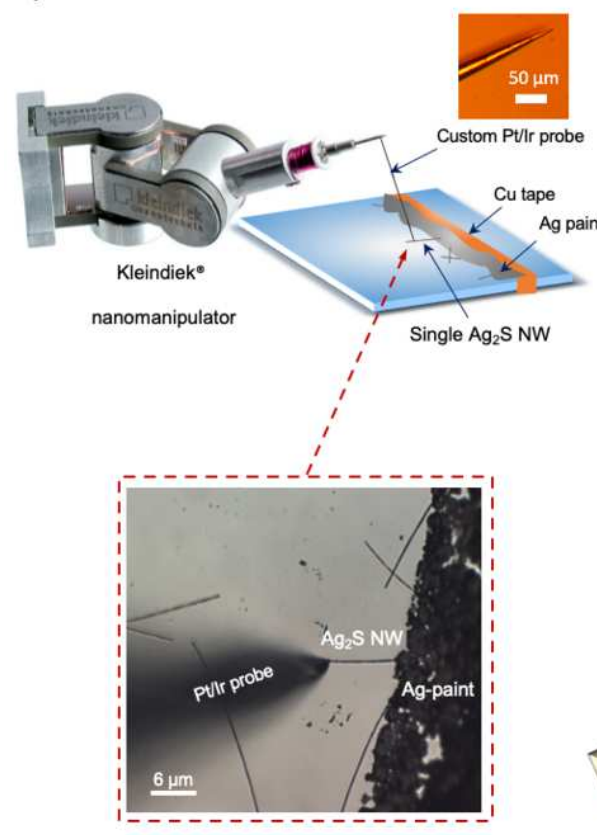

b)

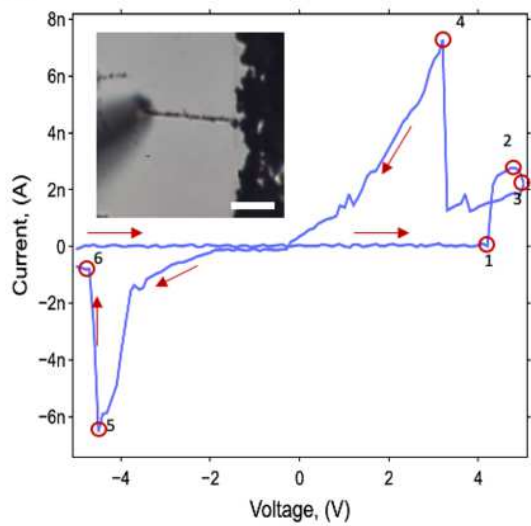

d)

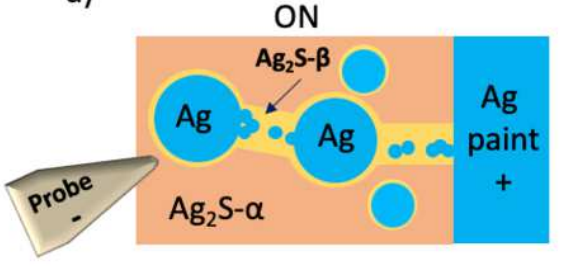

c)

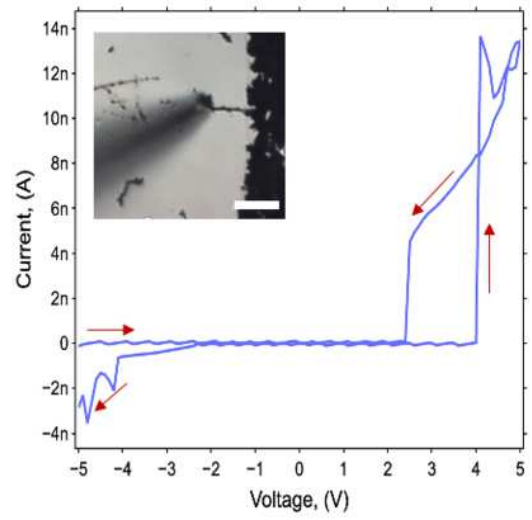

e)

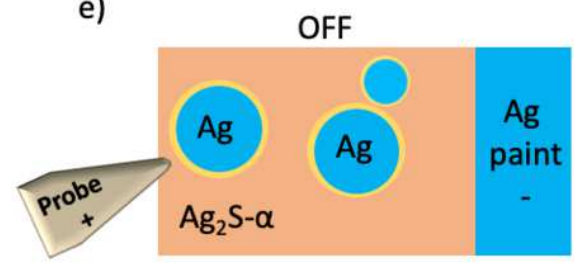

Figure 2. Resistive switching of a single $\mathrm{Ag}_{2} \mathrm{~S}$ NW. a) A nanomanipulator (Kleindiek Nanotechnik, GmbH) with a custom-made Pt/Ir 80-20 electrode contacting a tip of a single Ag NW exposed on a microscope slide with another tip submerged under Ag paint. b) A microscopic image and IV characteristic of a single $20 \mu \mathrm{m}$ long $\mathrm{Ag}_{2} \mathrm{~S} \mathrm{NW}_{\mathrm{N} d e r}$ a negative-positive-negative triangular-shaped voltage cycle. c) IV characteristics of the same $\mathrm{Ag}_{2} \mathrm{~S} \mathrm{NW}$ as in b) after it was shortened to $10 \mu \mathrm{m}$. d) Conducting (ON) state of the model of RS in a single $\mathrm{Ag}_{2} \mathrm{~S} \mathrm{NW}$ with scattered Ag nanocrystal inclusions and $\mathrm{Ag}_{2} \mathrm{~S}-\beta$ nanobridges in $\mathrm{Ag}_{2} \mathrm{~S}-\alpha$ phase (adapted from Xu et al. ${ }^{24}$ ). e) Non conducting (OFF) state of the model.

There are several noticeable features in the IV characteristic of Figure 2 (b). The 1st quadrant of the IV characteristics in Figure 2 (b) shows a negligibly small current until a sharp peak at $2.4 \mathrm{nA}$ slightly above $4 \mathrm{~V}$ (1st red circle), followed by a subsequent reduction of the current to $2 \mathrm{nA}$ at $5 \mathrm{~V}$ (2nd red circle). As voltage started to decrease during the reverse loop, the current decreased to $1 \mathrm{nA}$ until after passing the $3.5 \mathrm{~V}$ mark, the current instantly increased to just below $8 \mathrm{nA}$ (4th red circle), designating devices $\mathrm{ON}$ state. As the polarity of the voltage sweep crossed negative value, the current in the 3rd quadrant of Figure 2 (b) still showed the device ON state reaching the peak near $-4.5 \mathrm{~V}$ and $-6.5 \mathrm{nA}$ (5th red circle) and rapidly dropping to $0.5 \mathrm{nA}$ at the end of the cycle at $-5 \mathrm{~V}$ mark (6th red circle).

In Figure 2 (c), the same $\mathrm{Ag}_{2} \mathrm{~S}$ wire's length was reduced to $\mathrm{L}=10 \mu \mathrm{m}$ and stimulated with the same triangular voltage bias as in Figure 2 (b), which exhibited a different RS pattern. As with longer wire in Figure 2 (b), hard RS took place at 4 V, the maximum current increased by nearly $75 \%$. In other experiments, the ON-state resistance was proportional to the length of the wire and was consistent with a wide range of lengths of the wires with an approximate value of $10 \mathrm{k} / \mathrm{nm}$ as discussed further. Thus the RS process is not restricted to a single location within the wire, instead, it is distributed across its entire length.

The pinched hysteresis of the switching shown in Figures 2 (b) and (c) can be well described by the model of RS in a thin film $\mathrm{TiO}_{2-\mathrm{x}}$ memristor ${ }^{21}$. Although this model reproduces the key features of memristive behavior, such as pinched hysteresis, it does not describe all RS regimes, particularly the stochastic reversal of the loop near the maximum voltage we observed in Figure 2 (b) point 2 and 3 and Figure 2 (c). The twisting of the loop is spontaneous and is likely caused by Joule heating that leads to partial breakdown of Ag filaments, formed within the argentite $\mathrm{Ag}_{2} \mathrm{~S}-\beta$ phase as first described by Liao et al. ${ }^{6}$.

Thermal and crystalline vibrations ${ }^{19,20}$ induce distortions in the conducting filaments and nano-islands that can break down and be measured in the conductivity instabilities. However, it is likely that these instabilities are partially compensated by the scattered nano-islands and more developed filaments, as is supported by the smoother current curve during the reversal of the bias voltage displayed in Figures 2 (b) and (c).

\subsection{Memristor model}

In the basic memristive model ${ }^{21}$, the memristance $\mathrm{M}$ of the thin film RS element with thickness $\mathrm{D}$ is calculated by Equation 1. 


$$
M=R_{O N} \frac{x(t)}{D}+R_{O F F}\left(1-\frac{x(t)}{D}\right)
$$

In equation $1, \mathrm{R}_{\mathrm{ON}}$ and $\mathrm{R}_{\mathrm{OFF}}$ correspond to memristor being in either a highly conducting $\mathrm{R}_{\mathrm{ON}}$ state or a low conducting $\mathrm{R}_{\mathrm{OFF}}$ state correspondingly or an intermediate state according to the $\mathrm{x}$ parameter. The $\mathrm{x}$ parameter is a state variable that describes the boundary of the distribution of dopants, such as oxygen vacancies in anionic devices such as $\mathrm{TiO}_{2-\mathrm{x}}$ or the effective length of the filaments grown on the cathode towards the anode in cationic memristive devices such as $\mathrm{Ag}_{2} \mathrm{~S}$ and which rate of filament length change is described by Equation 2.

$$
\frac{d x}{d t}=\mu \frac{R_{O N}}{D} I F(x)
$$

The $\mathrm{Ag}_{2} \mathrm{~S}$ NWs' dimensions used in the experiment had an average diameter of $120 \mathrm{~nm}$ and an average length of 20 $\mu \mathrm{m}$. Therefore, different degrees of sulfurization will produce large variability of resistances of the $\mathrm{Ag}_{2} \mathrm{~S}$ wires even for the same length as a function of concentration and distribution of randomly scattered islands of argentite $\mathrm{Ag}_{2} \mathrm{~S}-\beta$ with $\mathrm{Ag}$ atoms inclusions and spontaneously formed $\mathrm{Ag}$ clusters in them under the influence of electric field bias. Furthermore, the $\mathrm{Ag}_{2} \mathrm{~S}-\beta$ pathways that penetrate the $\mathrm{Ag}_{2} \mathrm{~S}-\alpha$ phase along the electric field not only have lower resistivity compared to $\mathrm{Ag}_{2} \mathrm{~S}-\alpha$ but also allow for rapid migration of $\mathrm{Ag}^{+}$ions under the influence of an electric field but also provide an environment for the formation of $\mathrm{Ag}$ nanocrystals from agglomerated $\mathrm{Ag}^{+}$ions or remnants of metallic filaments as shown in Figures 2 (d) and (e) ${ }^{24}$.

Equations 3 and 4 introduce the $\mathrm{R}_{\mathrm{ON}}$ function that depends on stochastic parameter $\delta$ that governs the filament breakdown shown in Figures 2 (d) and (e). The parameter $\delta$ represents the volume fraction of metallic nano-islands and is constrained by equations 5 and 6 . Similar to $\mathrm{x}$ in the basic memristor model Equations 1 and 2, the unitless $\omega$ parameter represents an effective normalized length of the conductive filaments $\mathrm{L}_{\mathrm{f}}$ within the wire relative to its actual length $\mathrm{L}$. Namely $\omega=\mathrm{L}_{\mathrm{f}} / \mathrm{L}$ and $\omega$ takes values between 0 and 1 . Note that while $\mathrm{L}$ denotes an actual length measured with a microscope, in the simulation we replaced it with an effective length variable $\mathrm{L}_{\mathrm{e}}$.

The stochastic parameter $\delta$ governs the magnitude of $\mathrm{R}_{\mathrm{ON}}$ and specifies the volume fraction of nano-islands and conductive filaments in the $\mathrm{Ag}_{2} \mathrm{~S}$ matrix. Due to the fluctuations caused by $\mathrm{Ag}^{+}$ion redox exchange and metastable atomic positions, the conductive channel's thickness will also fluctuate unpredictably ${ }^{19,42}$, and hence can be modeled with a stochastic process described by Equations 5 and 6 with normally distributed noise with standard deviation parameter $\sigma$. Metallic Ag nano-clusters are formed and reorganized spontaneously within the volume of the $\mathrm{Ag}_{2} \mathrm{~S}$ phase due to the heat and electric field-induced relocation of $\mathrm{Ag}^{+}$ions in $3 \mathrm{D}$ volume $\mathrm{e}^{23,35}$. Therefore, percolation theory is used to connect the conductivity in the ON state via the $\mathrm{R}_{\mathrm{ON}}$ variable with the volume fraction of $\mathrm{Ag}$ nano-islands in the mixture of $\mathrm{Ag}_{2} \mathrm{~S}-\alpha$ and $\mathrm{Ag}_{2} \mathrm{~S}-\beta$ phases and thus is described by the power-law in Equation 5. Determination of the percolation threshold is a mundane task. Thus in the simulator, we set $\delta=\delta$ - $\delta_{0}$ where $\delta$ can take only positive values or zero.

Thus in the model high concentration of scattered nano-islands will produce a low $\mathrm{R}_{\mathrm{ON}}$ value and a low effective length $\mathrm{L}$ of the wire for fixed mobility $\mu$ found in the literature ${ }^{23,43}$. On the other hand, a low concentration of scattered islands will result in a high magnitude of $\mathrm{R}_{\mathrm{ON}}$ and effective length of the wire $\mathrm{L}_{\mathrm{e}}$ close to observed $\mathrm{L}$.

$$
\begin{aligned}
& V=\left(R_{O N}(\delta) \omega+R_{O F F}(1-\omega)\right) I, \text { where } R_{O F F}=L \rho_{O F F}, \text { and } R_{O N}=L \rho_{O N} \\
& \frac{d \omega}{d t}=\mu \frac{R_{O N}}{L^{2}} I F(\omega) \\
& R_{O N}(\delta)=L \rho_{O N}\left(\delta-\delta_{0}\right)^{-\beta} \\
& \delta=\delta_{\min }+\mathscr{N}\left(0, \sigma^{2}\right), \text { where, } \delta_{0} \leq \delta_{\min }=R_{O N \max }^{-\frac{1}{\beta}}+\delta_{0} \leq \delta \leq \delta_{\max }=R_{O N \min }^{-\frac{1}{\beta}}+\delta_{0}
\end{aligned}
$$

To test our hypothesis of spontaneous $\mathrm{R}_{\mathrm{ON}}$ update, we simulated a memristive nanowire described by equations 3 to 6 with the CircuitSymphony circuit simulator ${ }^{44}$ with the parameters of the following simulations. In equations 3 to 6 , the magnitudes of $\mathrm{R}_{\mathrm{ON}}$ and $\mathrm{R}_{\mathrm{OFF}}$ are proportional to the length of the $\mathrm{Ag}_{2} \mathrm{~S} \mathrm{NW}$ with the $\rho_{O F F}$ and $\rho_{O N}$ multiplier. To avoid an explicit 


\begin{tabular}{|l|l|}
\hline Parameter & Value \\
\hline$\rho_{\text {ON }}$ (linear resistivity in ON state) & $4.75 \times 10^{3} \frac{\Omega}{\mathrm{nm}}$ \\
$\rho_{\text {OFF }}$ (linear resistivity in OFF state) & $4.75 \times 10^{6} \frac{\Omega}{\mu \mathrm{m}}$ \\
$\omega$ (initial relative length of Ag filament) & 0 \\
$L$ (actual length of the wire) & $20 \mu \mathrm{m}$ \\
$L_{e}$ (effective length of the wire) & 8 to $12 \mu \mathrm{m}$ \\
$\mu$ (Ag ${ }^{+}$ion mobility) & $1 \times 10^{3}$ to $8 \times 10^{3} \frac{\mu \mathrm{m}^{2}}{\mathrm{sV}}$ \\
$\sigma$ (noise factor) & 20 to 50 \\
$R_{\text {ONmin }}$ (minimum resistance factor) & 0.5 \\
$R_{\text {ONmax }}$ (maximum resistance factor) & 5 \\
$\beta$ (percolation exponent) & 1.1 \\
Simulator Integration Step & $0.5 \mu \mathrm{s}$ \\
\hline
\end{tabular}

Table 1. Simulation parameters for stochastic memristor model in Figure 3.

definition of the minimum and maximum resistivity values in the ON state, we defined the boundary volume fractions $\delta_{\min }$ and $\delta_{\max }$ that confine the overall changes of the resistance. $\mathrm{R}_{\mathrm{ONmin}}$ and $\mathrm{R}_{\mathrm{ONmax}}$ are used as multipliers to the fixed value of resistivity $\rho$ on of the device in the ON state, to define the magnitude of $\mathrm{R}_{\mathrm{ON}}$ and satisfy the following inequality $\mathrm{R}_{\mathrm{ONmin}}<$ $\mathrm{R}_{\mathrm{ONmax}}$ that defines the boundary of fluctuations of $\mathrm{R}_{\mathrm{ON}}$.

Thus at the percolation threshold, when the volume fraction of Ag filaments $\delta$ is at its minimum, namely $\delta=\delta_{\min }>\delta_{0}$, the $\mathrm{R}_{\mathrm{ON}}$ will be at its maximum value, $\mathrm{R}_{\mathrm{ON}}=\rho_{O N} \mathrm{~L}\left(\delta_{\text {min }}-\delta_{0}\right)^{-\beta}=\mathrm{R}_{\mathrm{ONmax}} \mathrm{L} \rho_{O N}$ and when the volume fraction of $\mathrm{Ag}$ nanocrystals is above percolation threshold at some maximum value $\delta=\delta_{\max }, \mathrm{R}_{\mathrm{ON}}=\rho_{O N} \mathrm{~L}\left(\delta_{\max }-\delta_{0}\right)^{-\beta}=\mathrm{R}_{\mathrm{ONmin}} \mathrm{L} \rho_{O N}$ will correspond to the minimum value of $\mathrm{R}_{\mathrm{ON}}$. In the simulation, the dynamics of $\delta$ follow a random walk process. In the relationship, $\beta$ is the percolation exponent for 3D systems and can take values between 1.3 to $3^{45}$.

The boundary check of $\delta$ is provided in Equation 6 and is used at each iteration step in the simulator to prevent an unconstrained drift. If at some iteration step, the new value of $\delta$ produces $\mathrm{R}_{\mathrm{ON}}$ below $\mathrm{R}_{\mathrm{ONmin}} \mathrm{L} \rho_{O N}$, then the value of $\delta$ will be set to be equal to the highest value of $\delta_{\max }$. On the other hand, if the new randomly assigned value of $\delta$ causes $\mathrm{R}_{\mathrm{ON}}$ to grow above $\mathrm{R}_{\mathrm{ONmax}} \mathrm{L} \rho_{O N}$ the value of $\delta$ will be replaced with the smallest magnitude at the lower boundary $\delta_{\min }$.

The experimental simulation results are shown in Figure 3 (a), where $16 \mu \mathrm{m}$ long $\mathrm{Ag}_{2} \mathrm{~S} \mathrm{NW}$ was stimulated with four positive and four negative triangular pulses with $10 \mathrm{~s}$ period. The memristor was modeled with the parameters listed in Table 1. The fitting was performed with Bayesian optimization provided by Optuna library ${ }^{46}$, over a set of hand-picked discrete values of the model parameters. The loss was calculated as a Euclidean distance, or root-mean-squared distance, between the laboratory data points and model output at a particular time step for the whole duration of the stimulation. Thus, each iteration was comprised of 50 independent measurements, after which the mean loss was the metric of performance of the model for the iteration. Figures 3 (c) shows time-lapse of the deformation of the wire caused by the flow of $\mathrm{Ag}^{+}$ions and their deposition near the Pt/Ir electrode. The snapshots were taken near the peak values of the input voltage during the first 40 seconds of the stimulation shown in Figures 3 (a) and (b).

Interestingly, fitted values were found close to the actual values reported in the literature. In particular, the mobility $\mu$ was found between $1 \times 10^{3}$ to $8 \times 10^{3} \mu \mathrm{m}^{2} /(\mathrm{sV})$ and was only slightly below the mobility experimentally observed for the cubic $\mathrm{Ag}_{2} \mathrm{~S}-\beta$ phase $\mu=15 \times 10^{3} \mu \mathrm{m}^{2} /(\mathrm{sV})$ at $\mathrm{T}=450 \mathrm{~K}$ (for comparison the $\mathrm{Ag}^{+}$mobility in the monoclinic phase of $\mathrm{Ag}_{2} \mathrm{~S}-\alpha$, is $\mu \sim 1$ $\mu \mathrm{m}^{2} /(\mathrm{sV})$ at $\left.\mathrm{T}=300 \mathrm{~K}\right)^{23,43}$. The difference could be attributed to the presence of different charge carriers, namely electrons and $\mathrm{Ag}^{+}$ions, and a mixture of acanthite and argentite phases ${ }^{35}$.

The evidence of the $\mathrm{Ag}$ nano-crystal formation in $\mathrm{Ag}_{2} \mathrm{~S} \mathrm{NW}$ is also supported by comparing switching times in thin films and long wires. In an acanthite, $\mathrm{Ag}_{2} \mathrm{~S}-\alpha$ wire model with an effective length of $16 \mu \mathrm{m}$, switching between ON/OFF states would take approximately 50 seconds at $3 \mathrm{~V}$ driving voltage potential due to relatively low mobility of $\mathrm{Ag}^{+}$ions in the phase, namely $\mu=0.5 \mu \mathrm{m}^{2} /(\mathrm{sV})^{23,43}$. However, in the experiment and the best-fitted parameters of the model to the experimental data of a single $\mathrm{Ag}_{2} \mathrm{~S}$ NW (Figure 3 (d) and Table 1), switching occurs within tens of seconds. The best-fitting was found only when the wire had a shorter effective length between $\mathrm{L}_{\mathrm{e}}=8 \mu \mathrm{m}$ and $\mathrm{L}_{\mathrm{e}}=12 \mu \mathrm{m}$, compared to actual $\mathrm{L}=16 \mu \mathrm{m}$, and supports the hypothesis of scattered nano-islands in the volume of a single $\mathrm{Ag}_{2} \mathrm{~S} \mathrm{NW}$ device. Thus the area between electrodes is filled with a mixture of high ionic mobility argentite, low ionic mobility acanthite, and islands of Ag inclusions. Since the model only exhibits stochastic current pattern in ON state, there is no stochastic pattern in the OFF state as can be noticed comparing currents during negative sweep in Figures 3 (a) and (d). The IV characteristics of the model with zeroed noise parameter is identical to characteristics of a conventional memristor model ${ }^{21}$ and is shown in Figure 3 (e).

The reduction of the effective length of the wire compared to the actual length, namely the fitted parameters of the model 


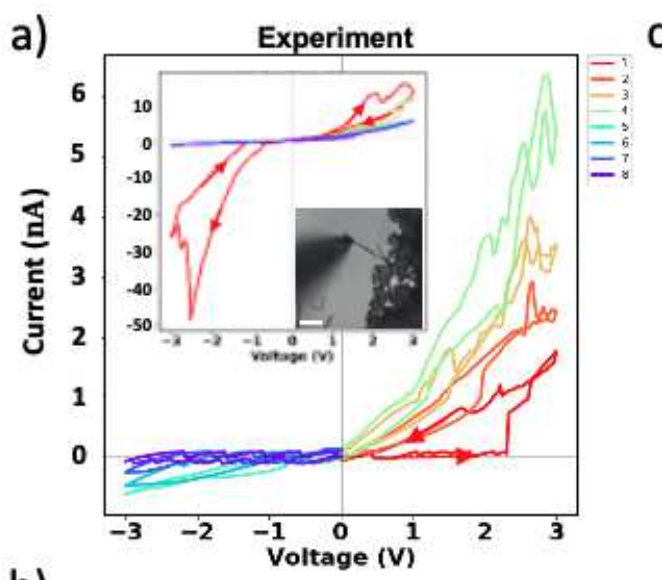

b)

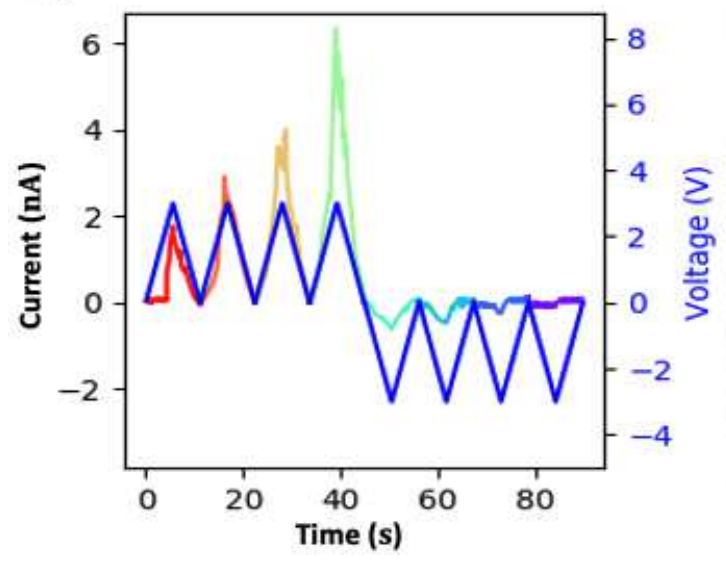

c)

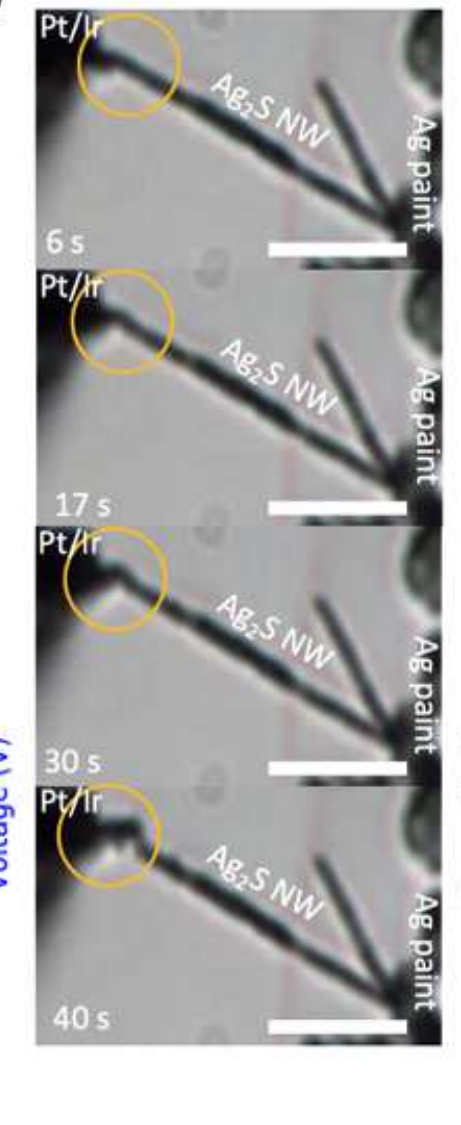

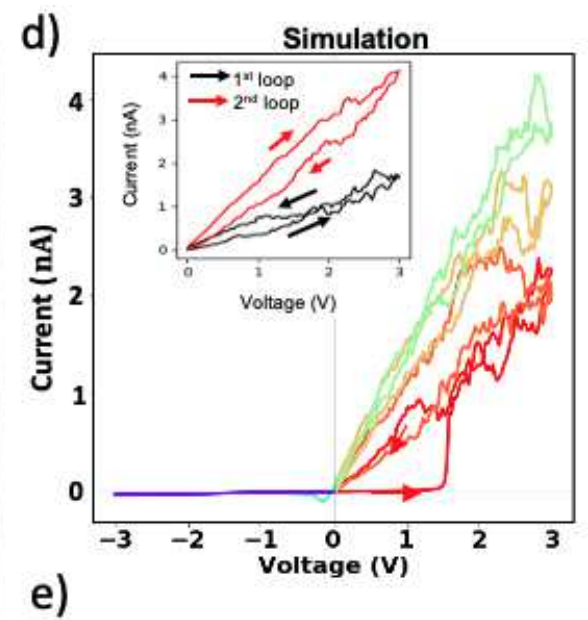

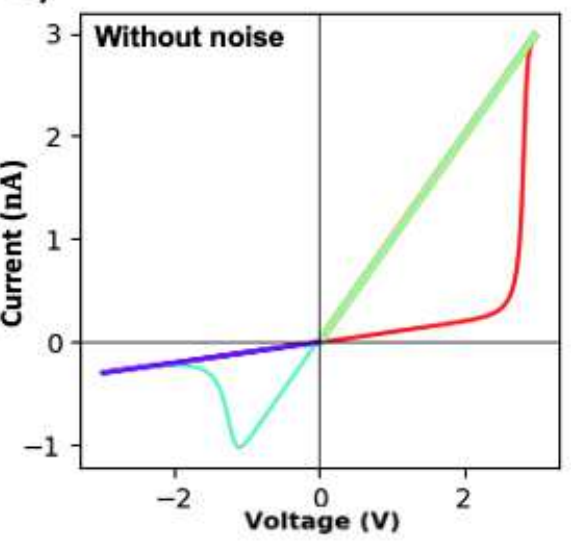

Figure 3. Measurement and modeling of stochastic RS of $\mathrm{Ag}_{2} \mathrm{~S}$ NW memristor. a) IV characteristics of a $16 \mu \mathrm{m}$ long $\mathrm{Ag}_{2} \mathrm{~S}$ NW stimulated first with four positive triangular pulses followed by four negative pulses (10 s period) showing OFF state (high resistance) at the negative bias and gradually reducing resistance in the ON state of the device. The inset IV plot showed spontaneous loop reversal when the wire was stimulated with alternating negative and positive triangular pulses with a $10 \mathrm{~s}$ period. The micrograph shows a single $\mathrm{Ag}_{2} \mathrm{~S} \mathrm{NW}$ contacted by the nanomanipulator. $\mathrm{b}$ ) Current response to triangular voltage stimulation of the $\mathrm{Ag}_{2} \mathrm{~S}$ NW. c) Time-lapse of the deformation caused by electromigration of $\mathrm{Ag}^{+}$ions in the $\mathrm{Ag}_{2} \mathrm{~S} \mathrm{NW}$ during the first 40s of the triangular voltage stimulation. d) Suboptimal simulation of the stochastic switching that replicates the behavior of the experimental data, with the proposed memristor model in equations 3 to 6 and the parameters from Table 1 . Inset in d) shows spontaneous loop reversal. e) Simulation of the RS with default parameters in Table 1 with zeroed noise parameter. All scale bars are $10 \mu \mathrm{m}$ long. 
showed an effective length range to be $\mathrm{L}_{\mathrm{e}}=8 \mu \mathrm{m}$ to $\mathrm{L}_{\mathrm{e}}=12 \mu \mathrm{m}$, which is less than the actual length of $\mathrm{L}=16 \mu \mathrm{m}$. The shortening of the wire, obtained from the fittings, also argues against the core-shell structure of the wire (i.e. nonexistence of a unit $\mathrm{Ag}$ core) and speaks in support of the fragmented organization with $\mathrm{Ag}$ inclusions within $\mathrm{Ag}_{2} \mathrm{~S} \mathrm{NW}$ that shortens its effective length. The inset in Figure 3 (a) shows the loop reversal in both 1st and 3rd quadrant during the stimulation of the previously set to $\mathrm{ON}$ state memristive $\mathrm{Ag}_{2} \mathrm{~S} \mathrm{NW}$ with alternating negative and positive triangular voltage pulses. During the first stimulation period, the loop goes clockwise. During the second portion, the loop is also clockwise, which signifies spontaneous (perhaps Joule heating-dominated) destruction of the conducting Ag filaments within the NW. Simulation results exhibit behavior similar to experimental data and are shown in Figure 3 (b). The probability of loop twisting and reversal can be controlled in the memristor model by changing $\mathrm{R}_{\mathrm{ONmin}}$ and $\mathrm{R}_{\mathrm{ONmax}}$ and noise factor $\sigma$.

\section{Discussion}

This work presented a simple method of measuring the electronic properties of individual nanowires with a nanomanipulator under an optical microscope. Based on the experimental IV characteristics of a single $\mathrm{Ag}_{2} \mathrm{~S} \mathrm{NW}$, we modified a basic memristor model with a resistivity of the memristor varying as a function of a volume fraction of conducting filaments that spontaneously create percolating pathways that result in noise and variability in the current. The model also reproduced key features of the experimental data, such as spontaneous loop reversal and loop twisting during voltage sweep, and suggests that further refinement could be achieved through an in-depth investigation of the percolative nature of resistive switching in a single NW. The results obtained in this work can be used to develop larger models of randomly self-assembled neuromorphic systems that naturally exhibit instability and noise.

In the presented model, the resistive switching mechanism was dependant on two-state variables $\omega$ and $\delta$. The length of the conductive Ag filament is described by variable $\omega$. The stochastic variable $\delta$ describes the decay and spontaneous creation of the conducting channel due to the random redox processes. Also, the variable $\delta$ represents the thickness of the formed conductive channels, the process that is governed by the percolation theory.

Despite the model's good performance and ability to obtain decent fit characteristics to experimental data, further refinement of the model with additional data, such as quantitative measurement of the $\mathrm{Ag}$ islands and temporal distribution of them from an in situ HRTEM during the RS, can significantly improve it.

The robustness of the model might also be increased by taking into account thermodynamic parameters of the NW, such as modeling Joule heating and Newton's cooling. The stochastic nature of the conductive filament creation and destruction in the $\mathrm{Ag}_{2} \mathrm{~S} \mathrm{NW}$ is an exciting property that mimics biological synapses. Biological synapses exhibit stochastic openings of ionic channels that cause unreliability of signal propagation through the synapse that are thought to play an essential role in the brain's energy conservation and learning ${ }^{47-49}$.

\section{Methods}

\subsection{Nanowire production}

First, the Ag NWs were produced with a simple polyol method ${ }^{31-33}$. Further, the NWs was sulfurized by ultrasonic dispersion of sulfur powders ( $\mathrm{S}$ ) in the $\mathrm{Ag} \mathrm{NW-EtOH}$ suspension (based on $\mathrm{Ag}$ and $\mathrm{S}$ stoichiometric ratio) at $\sim 60^{\circ} \mathrm{C}$ for $5,7,10$ minutes to produce light brown, dark brown, and black suspension, respectively ${ }^{50,51}$.

\subsection{Electronic measurements}

To create a single $\mathrm{Ag}_{2} \mathrm{~S}$ wire device, a single droplet of dark brown $\mathrm{Ag}_{2} \mathrm{~S}$ NW suspension in Ethanol (3.8 $\left.\mu \mathrm{g} / \mathrm{ml}\right)$, was drop cast on top of a clean microscope slide and placed on a hot plate to allow ethanol evaporation. After solvent evaporation, a sparse nonconducting network of NWs is formed on the microscope slide. A thin layer of Ag paint was deposited to partially cover the exposed NWs.

\section{References}

1. Jeong, D. S., Kim, K. M., Kim, S., Choi, B. J. \& Hwang, C. S. Memristors for Energy-Efficient New Computing Paradigms. Adv. Electron. Mater. 2, 1600090, DOI: 10.1002/aelm.201600090 (2016).

2. Camuñas-Mesa, L., Linares-Barranco, B. \& Serrano-Gotarredona, T. Neuromorphic Spiking Neural Networks and Their Memristor-CMOS Hardware Implementations. Materials 12, 2745, DOI: 10.3390/ma12172745 (2019).

3. Choi, S., Sheridan, P. \& Lu, W. D. Data Clustering using Memristor Networks. Sci. Reports 5, DOI: 10.1038/srep10492 (2015).

4. Merolla, P. A. et al. A million spiking-neuron integrated circuit with a scalable communication network and interface. Science 345, 668-673, DOI: 10.1126/science.1254642 (2014). 
5. Liang, C., Terabe, K., Hasegawa, T. \& Aono, M. Resistance switching of an individual Ag2S/Ag nanowire heterostructure. Nanotechnology 18, 485202, DOI: 10.1088/0957-4484/18/48/485202 (2007).

6. Liao, Z.-M., Hou, C., Zhang, H.-Z., Wang, D.-S. \& Yu, D.-P. Evolution of resistive switching over bias duration of single Ag2S nanowires. Appl. Phys. Lett. 96, 203109, DOI: 10.1063/1.3432665 (2010).

7. Zhuge, F. et al. Mechanism for resistive switching in chalcogenide-based electrochemical metallization memory cells. AIP Adv. 5, 057125, DOI: 10.1063/1.4921089 (2015).

8. Ciocchini, N. et al. Bipolar switching in chalcogenide phase change memory. Sci. Reports 6, DOI: 10.1038/srep29162 (2016).

9. Pi, C., Ren, Y. \& Chim, W. K. Investigation of bipolar resistive switching and the time-dependent SET process in silver sulfide/silver thin films and nanowire array structures. Nanotechnology 21, 085709, DOI: 10.1088/0957-4484/21/8/085709 (2010).

10. Wagenaar, J. J. T., Morales-Masis, M. \& van Ruitenbeek, J. M. Observing "quantized" conductance steps in silver sulfide: Two parallel resistive switching mechanisms. J. Appl. Phys. 111, 014302, DOI: 10.1063/1.3672824 (2012).

11. Sillin, H. O. et al. A theoretical and experimental study of neuromorphic atomic switch networks for reservoir computing. Nanotechnology 24, 384004, DOI: 10.1088/0957-4484/24/38/384004 (2013).

12. Lilak, S. et al. Spoken Digit Classification by In-Materio Reservoir Computing With Neuromorphic Atomic Switch Networks. Front. Nanotechnol. 3, DOI: 10.3389/fnano.2021.675792 (2021).

13. Lutz, C., Hasegawa, T. \& Chikyow, T. Ag2S atomic switch-based 'tug of war' for decision making. Nanoscale 8, 14031-14036, DOI: 10.1039/c6nr00690f (2016).

14. Ielmini, D. \& Ambrogio, S. Emerging neuromorphic devices. Nanotechnology 31, 092001, DOI: 10.1088/1361-6528/ ab554b (2019).

15. Fu, K. et al. Reservoir Computing with Neuromemristive Nanowire Networks. In 2020 International Joint Conference on Neural Networks (IJCNN), DOI: 10.1109/ijenn48605.2020.9207727 (IEEE, 2020).

16. del Valle, J., Ramírez, J. G., Rozenberg, M. J. \& Schuller, I. K. Challenges in materials and devices for resistive-switchingbased neuromorphic computing. J. Appl. Phys. 124, 211101, DOI: 10.1063/1.5047800 (2018).

17. Lu, W. \& Lieber, C. M. Nanoelectronics from the bottom up. Nat. Mater. 6, 841-850, DOI: 10.1038/nmat2028 (2007).

18. Du, C. et al. Reservoir computing using dynamic memristors for temporal information processing. Nat. Commun. 8, DOI: 10.1038/s41467-017-02337-y (2017).

19. Sánta, B. et al. Universal $1 / \mathrm{f}$ type current noise of Ag filaments in redox-based memristive nanojunctions. Nanoscale 11, 4719-4725, DOI: 10.1039/c8nr09985e (2019).

20. Sánta, B. et al. Noise Tailoring in Memristive Filaments. ACS Appl. Mater. \& Interfaces 13, 7453-7460, DOI: 10.1021/ acsami.0c21156 (2021).

21. Strukov, D. B., Snider, G. S., Stewart, D. R. \& Williams, R. S. The missing memristor found. Nature 453, 80-83, DOI: 10.1038/nature06932 (2008).

22. Banerjee, S., Bhattacharya, S. \& Chakravorty, D. Resistivity Hysteresis of Ag2S Nanocomposites. The J. Phys. Chem. C 111, 13410-13413, DOI: 10.1021/jp073814b (2007).

23. Simonnin, P., Sassi, M., Gilbert, B., Charlet, L. \& Rosso, K. M. Phase Transition and Liquid-like Superionic Conduction in Ag2S. The J. Phys. Chem. C 124, 10150-10158, DOI: 10.1021/acs.jpcc.0c00260 (2020).

24. Xu, Z., Bando, Y., Wang, W., Bai, X. \& Golberg, D. Real-Time In Situ HRTEM-Resolved Resistance Switching of Ag2S Nanoscale Ionic Conductor. ACS Nano 4, 2515-2522, DOI: 10.1021/nn100483a (2010).

25. Cava, R., Reidinger, F. \& Wuensch, B. Single-crystal neutron diffraction study of the fast-ion conductor -Ag2S between 186 and $325^{\circ}$ C. J. Solid State Chem. 31, 69-80, DOI: 10.1016/0022-4596(80)90009-2 (1980).

26. He, X. et al. Memristive properties of hexagonal WO3 nanowires induced by oxygen vacancy migration. Nanoscale Res. Lett. 8, DOI: 10.1186/1556-276x-8-50 (2013).

27. Xiao, M., Musselman, K. P., Duley, W. W. \& Zhou, N. Y. Resistive Switching Memory of TiO2 Nanowire Networks Grown on Ti Foil by a Single Hydrothermal Method. Nano-Micro Lett. 9, DOI: 10.1007/s40820-016-0116-2 (2016).

28. Milano, G. et al. Self-limited single nanowire systems combining all-in-one memristive and neuromorphic functionalities. Nat. Commun. 9, DOI: 10.1038/s41467-018-07330-7 (2018). 
29. Wang, Z. et al. Memristors with diffusive dynamics as synaptic emulators for neuromorphic computing. Nat. Mater. 16, 101-108, DOI: 10.1038/nmat4756 (2016).

30. Ohno, T. \& Hasegawa, T. Observation of a Ag protrusion on a Ag $2 \mathrm{~S}$ island using a scanning tunneling microscope. Results Phys. 5, 182-183, DOI: 10.1016/j.rinp.2015.08.004 (2015).

31. Zhang, K., Du, Y. \& Chen, S. Sub $30 \mathrm{~nm}$ silver nanowire synthesized using KBr as co-nucleant through one-pot polyol method for optoelectronic applications. Org. Electron. 26, 380-385, DOI: 10.1016/j.orgel.2015.08.008 (2015).

32. Chen, C. et al. Study on the growth mechanism of silver nanorods in the nanowire-seeding polyol process. Mater. Chem. Phys. 107, 13-17, DOI: 10.1016/j.matchemphys.2007.06.048 (2008).

33. Andrés, L. J. et al. Rapid synthesis of ultra-long silver nanowires for tailor-made transparent conductive electrodes: proof of concept in organic solar cells. Nanotechnology 26, 265201, DOI: 10.1088/0957-4484/26/26/265201 (2015).

34. Levard, C. et al. Sulfidation Processes of PVP-Coated Silver Nanoparticles in Aqueous Solution: Impact on Dissolution Rate. Environ. Sci. \& Technol. 45, 5260-5266, DOI: 10.1021/es2007758 (2011).

35. Hebb, M. H. Electrical Conductivity of Silver Sulfide. The J. Chem. Phys. 20, 185-190, DOI: 10.1063/1.1700165 (1952).

36. Motte, L. \& Urban, J. Silver Clusters on Silver Sulfide Nanocrystals: Synthesis and Behavior after Electron Beam Irradiation. The J. Phys. Chem. B 109, 21499-21501, DOI: 10.1021/jp0542322 (2005).

37. Gusev, A. \& Sadovnikov, S. Structure and properties of nanoscale Ag 2 S/Ag heterostructure. Mater. Lett. 188, 351-354, DOI: 10.1016/j.matlet.2016.11.111 (2017).

38. Nayak, A. et al. Rate-Limiting Processes Determining the Switching Time in a Ag2S Atomic Switch. The J. Phys. Chem. Lett. 1, 604-608, DOI: 10.1021/jz900375a (2010).

39. Terabe, K., Nakayama, T., Hasegawa, T. \& Aono, M. Formation and disappearance of a nanoscale silver cluster realized by solid electrochemical reaction. J. Appl. Phys. 91, 10110, DOI: 10.1063/1.1481775 (2002).

40. Zhang, M. \& Lian, X. Rapid Fabrication of High-Aspect-Ratio Platinum Microprobes by Electrochemical Discharge Etching. Materials 9, 233, DOI: 10.3390/ma9040233 (2016).

41. Khan, Y., Al-Falih, H., Zhang, Y., Ng, T. K. \& Ooi, B. S. Two-step controllable electrochemical etching of tungsten scanning probe microscopy tips. Rev. Sci. Instruments 83, 063708, DOI: 10.1063/1.4730045 (2012).

42. Gubicza, A. et al. Asymmetry-induced resistive switching in Ag-Ag2S-Ag memristors enabling a simplified atomic-scale memory design. Sci. Reports 6, DOI: 10.1038/srep30775 (2016).

43. Allen, R. L. \& Moore, W. J. Diffusion of Silver in Silver Sulfide. The J. Phys. Chem. 63, 223-226, DOI: 10.1021/ j150572a021 (1959).

44. Frick, N. \& LaBean, T. nfrik/CircuitSymphony: rel-osx-v9.0.1, DOI: 10.5281/zenodo.4545814 (2021).

45. Kim, Y. J. et al. Electrical conductivity of chemically modified multiwalled carbon nanotube/epoxy composites. Carbon 43, 23-30, DOI: 10.1016/j.carbon.2004.08.015 (2005).

46. Akiba, T., Sano, S., Yanase, T., Ohta, T. \& Koyama, M. Optuna: A Next-generation Hyperparameter Optimization Framework (2019). 1907.10902.

47. Stevens, C. F. \& Zador, A. M. Input synchrony and the irregular firing of cortical neurons. Nat. Neurosci. 1, 210-217, DOI: $10.1038 / 659$ (1998).

48. Rusakov, D. A., Savtchenko, L. P. \& Latham, P. E. Noisy Synaptic Conductance: Bug or a Feature? Trends Neurosci. 43, 363-372, DOI: 10.1016/j.tins.2020.03.009 (2020).

49. Harris, J. J., Jolivet, R. \& Attwell, D. Synaptic Energy Use and Supply. Neuron 75, 762-777, DOI: 10.1016/j.neuron.2012. 08.019 (2012).

50. Zhang, S. et al. Facile preparation of Ag-Ag2S hetero-dendrites with high visible light photocatalytic activity. J. Mater. Sci. 53, 6482-6493, DOI: 10.1007/s10853-018-2032-y (2018).

51. Xiong, J. et al. Ambient synthesis of a multifunctional 1D/2D hierarchical Ag-Ag2S nanowire/nanosheet heterostructure with diverse applications. CrystEngComm 18, 930-937, DOI: 10.1039/c5ce02134k (2016). 


\section{Acknowledgements}

We thank Dr. Lorenz Lechner (Kleindiek Nanotechnik $\mathrm{GmbH}$ ) for providing MM3A nanomanipulator. The authors acknowledge the financial support for this project from the US National Science Foundation grant NSF-CISE-CCF 1748459. This work was performed in part at the Analytical Instrumentation Facility (AIF) at North Carolina State University, which is supported by the State of North Carolina and the National Science Foundation (award number ECCS-1542015). This work made use of instrumentation at AIF acquired with support from the National Science Foundation (DMR-1726294). The AIF is a member of

the North Carolina Research Triangle Nanotechnology Network (RTNN), a site in the National Nanotechnology Coordinated Infrastructure (NNCI).

\section{Author contributions statement}

N.F., M.H., and T.L. conceived and designed the experiments, N.F., M.H, and M.G. conducted the experiments, M.H., N.F., D.G., and T.L. analyzed the data, N.F., M.H., D.G. and T.L. wrote the paper. All authors reviewed the manuscript. All authors reviewed the manuscript.

\section{Additional information}

Competing financial interests: The authors declare no competing financial interests. 